\title{
Anatomy and physiology of the enteric nervous system
}

\author{
M Costa, S J H Brookes, G W Hennig
}

The enteric nervous system (ENS) is a quasi autonomous part of the nervous system and includes a number of neural circuits that control motor functions, local blood flow, mucosal transport and secretions, and modulates immune and endocrine functions. Although these functions operate in concert and are functionally interlinked, it is useful to consider the neural circuits involved in each separately. ${ }^{1}$ This short summary will concentrate mainly on the neural circuits involved in motor control. $^{2}$ The enteric neural circuits are composed of enteric neurones arranged in networks of enteric ganglia connected by interganglionic strands. Most enteric neurones involved in motor functions are located in the myenteric plexus with some primary afferent neurones located in the submucous plexus. As in all nervous systems involved in sensory-motor control, the ENS comprises primary afferent neurones, sensitive to chemical and mechanical stimuli, interneurones and motorneurones that act on the different effector cells including smooth muscle, pacemaker cells, blood vessels, mucosal glands, and epithelia, and the distributed system of intestinal cells involved in immune responses and endocrine and paracrine functions.

The digestive tract is unique among internal organs because it is exposed to a large variety of physicochemical stimuli from the external world in the form of ingested food. As a consequence, the intestine has developed a rich repertoire of coordinated movements of its muscular apparatus to ensure the appropriate mixing and propulsion of contents during digestion, absorption, and excretion. The oro-aboral transit of the intestinal contents can be regarded as a form of adaptive locomotion that occurs over a wide range of spatial and temporal domains. ${ }^{3}$ The movements of the intestine are the result of interaction of the neural apparatus and the muscular apparatus. ${ }^{4}$

The muscular apparatus is organised in muscle layers made up of large collections of smooth muscle cells interconnected electrically via gap junctions to operate as larger functional mechanical units. The membrane potential of smooth muscle is driven to oscillate (slow waves) by a syncytial network of pacemaker cells (interstitial cells of Cajal) probably also via gap junctions. ${ }^{5}$ As the action potentials of the smooth muscles, and thus their associated muscle contraction, do not appear to propagate over long distances, the coordination of muscle activity over long distances is highly dependent on the spatiotemporal patterns of the slow wave generated by the pacemaker networks. The myogenic patterns of activity can support pro-

Correspondence to: Professor M Costa. marcello.costa@flinders.edu.au and in the duodenum. It is on this spontaneously active muscular apparatus that the enteric motor circuits play their roles in shaping different motor patterns.

The neural apparatus is composed of a large number of enteric neurones that can be identified according to their location, neurochemistry, shape, projections, proportions, connections, and function. After intensive research from several laboratories over the past two decades, a full description of all functional classes of enteric neurones has been recently achieved in the guinea pig small intestine (fig 1). ${ }^{6}$ The strategy included the development of methods combining immunohistochemistry, electrophysiology, retrograde tracing, neuronal filling, lesion techniques, and pharmacological analysis.

\section{The enteric neurones}

PRIMARY AFFERENT NEURONES

Primary afferent neurones (also termed enteric primary afferent neurones (EPANs) or intrinsic primary afferent neurones (IPANs)) are present in both myenteric and submucous ganglia. They respond to luminal chemical stimuli, to mechanical deformation of the mucosa, and to radial stretch and muscle tension. It is not yet clear whether epithelial cells such as enterochromaffin cells release substances, for instance serotonin, in response to chemical or mechanical stimuli, to activate the endings of the primary afferent neurones. ${ }^{7}$ They represent about $30 \%$ of myenteric neurones and $14 \%$ of submucosal neurones, have a distinct Dogiel type II shape and have a long after hyperpolarisation following action potentials. All of these neurones project to the villi and branch within the submucous and myenteric ganglia locally. A proportion of these neurones $(10 \%$ of primary afferent neurones) also have long descending projections to aboral myenteric ganglia. ${ }^{8}$ They receive slow synaptic input (probably mediated by tachykinins) from other primary afferent neurones to form reciprocally innervated networks. They project circumferentially to synapse with myenteric ascending interneurones, descending interneurones, longitudinal muscle motorneurones, excitatory circular muscle motorneurones, and inhibitory circular muscle motorneurones. It is likely that different subpopulations are connected separately, with ascending and descending pathways.

Abbreviations used in this paper: ENS, enteric nervous system; EPANs, enteric primary afferent neurones; IPANs, intrinsic primary afferent neurones; ChAT, choline acetyltransferase; VIP, vasoactive intestinal peptide; NOS, nitric oxide synthase. 
EXCITATORY CIRCULAR MUSCLE

MOTORNEURONES

These represent the final motor output to the circular muscle (14\%), have a Dogiel type I shape, receive fast nicotinic and probably slow synaptic input from local primary afferent neurones, and from the only class of cholinergic ascending interneurones. They also appear to receive excitatory inputs from descending interneurones. They project to the circular muscle where they form a denser arrangement of nerve endings in the deep muscular plexus. They use acetylcholine and tachykinins as transmitters acting directly on smooth muscle and possibly indirectly via the network of interstitial cells in the deep muscular plexus. ${ }^{9-11}$

INHIBITORY CIRCULAR MUSCLE MOTORNEURONES These Dogiel type I neurones (17\%) receive fast nicotinic inputs from primary afferent neurones and non-cholinergic inputs from the long descending primary afferent neurones. They project to the circular muscle where their axons are intimately associated with those of the excitatory motorneurones in the deep muscular plexus. They use multiple mechanisms of inhibitory transmission including nitric oxide, adenosine triphosphate, and the peptides vasoactive intestinal peptide (VIP) and pituitary activating cyclic AMP peptide acting directly on smooth muscle or indirectly via interstitial cells. ${ }^{911}$

LONGITUDINAL MUSCLE MOTORNEURONES This relatively large class $(25 \%)$ of small neurones with short projections to the longitudinal muscle receive synaptic inputs from the enteric primary afferent neurones and from ascending and descending pathways. ${ }^{12}$
ASCENDING INTERNEURONES

This small $(5 \%)$ but most important class of enteric neurones belongs to the Dogiel type I morphology, and receives fast synaptic inputs from other ascending interneurones which form a chain of ascending excitation. They also receive fast nicotinic and slow synaptic inputs from enteric primary afferent neurones. They project orally within the myenteric plexus to synapse with the final excitatory circular muscle motor neurones via fast nicotinic and noncholinergic slow synaptic inputs. They contain not only the enzyme for the synthesis of acetylcholine but also tachykinins and opioid peptides. $^{13}$

\section{DESCENDING INTERNEURONES}

There are several classes of descending interneurones that comprise about $7 \%$ of the total. ${ }^{614}$ Three of these are probably cholinergic as they contain the enzyme for the synthesis of acetylcholine, choline acetyltransferase (ChAT). Each differs in their neurochemistry. Somatostatin and ChAT containing descending interneurones $(4 \%)$ have a filamentous shape, receive fast and slow synaptic inputs mainly from non-primary afferent neurones, and form a chain of interconnected interneurones synapsing with other somatostatin neurones and with other myenteric and submucous neurones. Serotonin and ChAT containing neurones $(2 \%)$ project aborally to other myenteric and submucosal neurones but not to inhibitory motorneurones. Whether these neurones use serotonin in addition to acetylcholine remains to be confirmed. Serotonin may act via fast ion channel gated receptors or via slow $\mathrm{G}$ protein linked receptors. Nitric oxide synthase (NOS), VIP, and ChAT

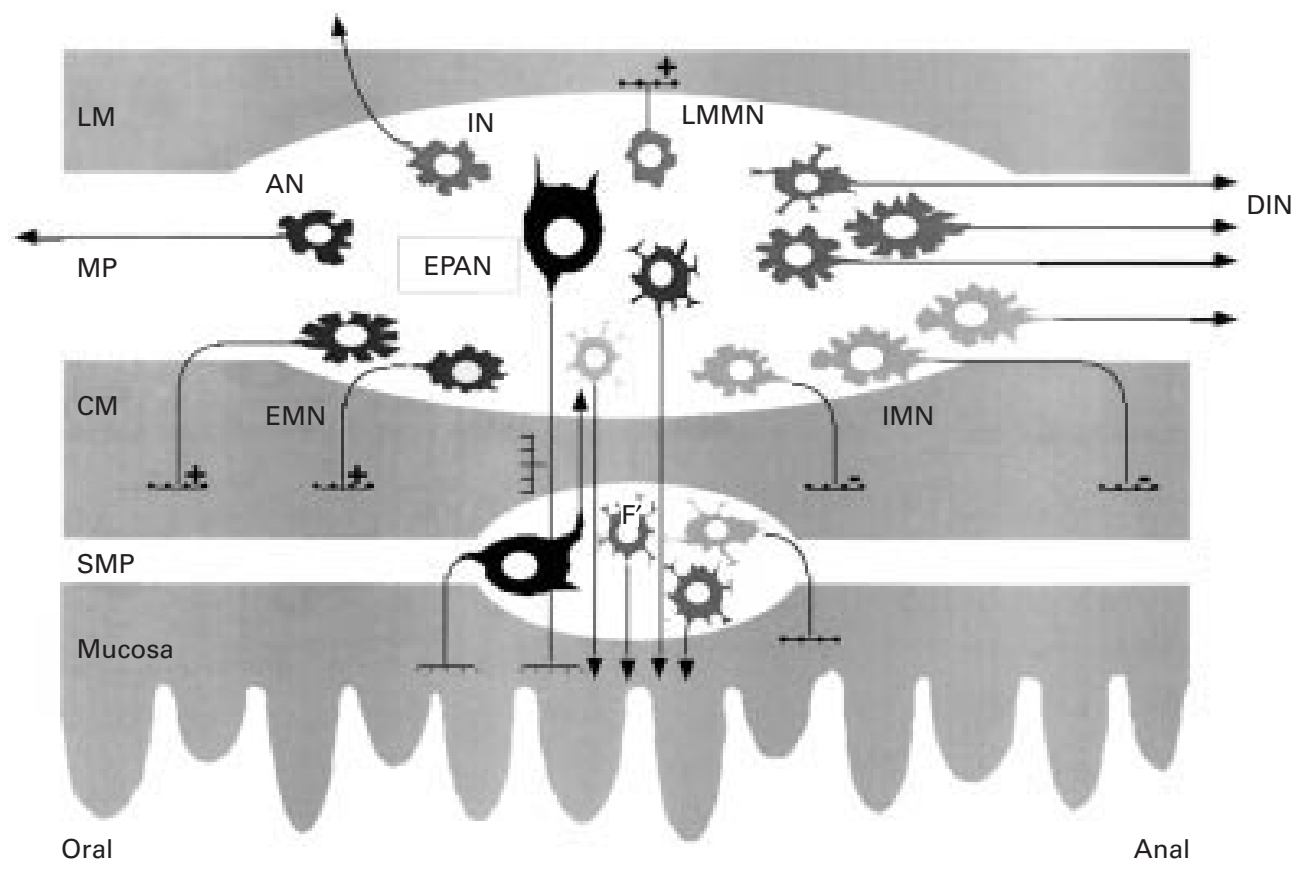

Figure 1 Classes of myenteric neurones. LM, longitudinal muscle; CM, circular muscle; MP, myenteric plexus; SMP, submucous plexus; $A N$, ascending neurones; IN, intestinofugal neurones; DIN, descending interneurones; EPAN, enteric primary afferent neurones; EMN, excitatory motorneurones; IMN, inhibitory motorneurones; LMMN, longitudinal motorneurones. The secretomotor and vasomotor neurones in the myenteric and submucous plexuses are not labelled (modified from Costa and colleagues ${ }^{6}$ ). 


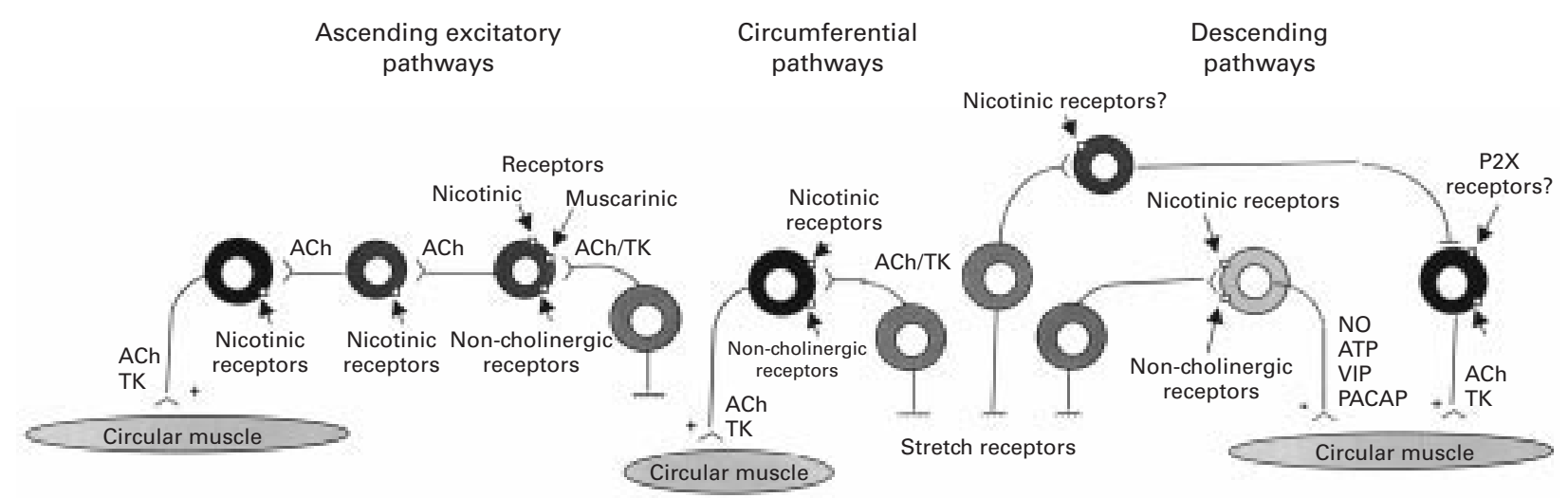

Figure 2 Polarised enteric motor pathways involved in control of intestinal motor activity. ACh, acetylcholine; TK, tachykinins; NO, nitric oxide; ATP, adenosine triphosphate; VIP, vasoactive intestinal peptide; PACAP, pituitary activating cyclic AMP peptide; P2X, subclass of purinergic receptors.

containing neurones also project aborally to synapse with other myenteric neurones. Neurones with NOS and VIP, but without ChAT, also project to other aboral myenteric and probably submucous ganglia. Whether these non-cholinergic interneurones use other fast synaptic transmitters such as adenosine triphosphate or glutamate remains to be established.

The dual projection of some of these interneurones to both myenteric and submucous ganglia represents the likely functional link between motor, secretory, and vasomotor pathways.

SECRETOMOTOR AND VASOMOTOR NEURONES

There are two small classes ( $1 \%$ each) of secretomotor neurones in the myenteric ganglia. One are cholinergic and the other noncholinergic containing VIP. They project to the mucosa. Neurones with a similar function and neurochemistry are also present in the submucous ganglia where they represent $32 \%$ and $42 \%$, respectively. Some of the VIP submucous neurones also project to the myenteric ganglia and may represent the basis for a functional connection between secretion and motility. The VIP secretomotor neurones receive inhibitory synaptic inputs from the extrinsic sympathetic neurones and from unidentified myenteric neurones. Most submucous neurones receive fast and slow synaptic inputs. A small submucous neurone class of submucous cholinergic neurones (12\%) project to the mucosa and to the local blood vessels.

INTESTINOFUGAL NEURONES

There is a small proportion of cholinergic neurones that receive fast synaptic inputs, and project from myenteric ganglia to the prevertebral ganglia.

OTHER GASTROINTESTINAL REGIONS

The remarkable polarities of enteric motorneurones and interneurones, revealed in the small intestine, extend also to the oesophagus, stomach, and large intestine, suggesting that it is a prominent and preserved feature of the arrangement of the enteric neural pathways. In the different regions there are also significant differences in the classes represented and in their neurochemical coding. For example, there are very few enteric primary afferent neurones of the Dogiel type II in the stomach.

\section{Enteric neural circuits}

The initial steps in the elucidation of the enteric neural circuits has been accomplished by using the classic approach of specific and localised stimuli and recording of the reflex responses. It was the demonstration of polarised responses to mechanical stimuli by Bayliss and Starling $(1899)^{15}$ that started the modern analysis of enteric reflex pathways. They postulated the existence of short ascending excitatory pathways and longer descending inhibitory pathways giving rise to the idea of "the law of the intestine". In recent years, analysis of such pathways has advanced significantly (fig 2). Thus there are ascending excitatory pathways that involve the EPANs, a chain of short ascending interneurones, and the final excitatory motorneurones to the circular muscle. The descending inhibitory pathways probably involve a different class of EPANs, with long anal projections connected to the final inhibitory motorneurone. There are also circumferential pathways that are activated by mechanical stimulation of the EPANs, which synapse with local inhibitory and excitatory motorneurones. There is also a descending excitatory pathway that involves mechanically sensitive EPANs and final excitatory motorneurones to the circular muscle. Whether there are descending interneurones in these pathways remain to be established. The reflex pathways involving motor responses of the longitudinal muscle have been poorly investigated. There are also reflex pathways involving the rare intestinofugal neurones, located in the myenteric ganglia, which synapse in the prevertebral ganglia with postganglionic sympathetic neurones projecting to the submucous and myenteric ganglia. These represent short intestino-intestinal inhibitory reflex pathways that, when activated, reduce both motor and secretomotor activity.

Similar mechanical and chemical stimuli are associated with secretomotor and vasomotor responses. The secretomotor and vasomotor neural pathways underlying these responses involve EPANs both in the submucous and myenteric ganglia and cholinergic and non- 


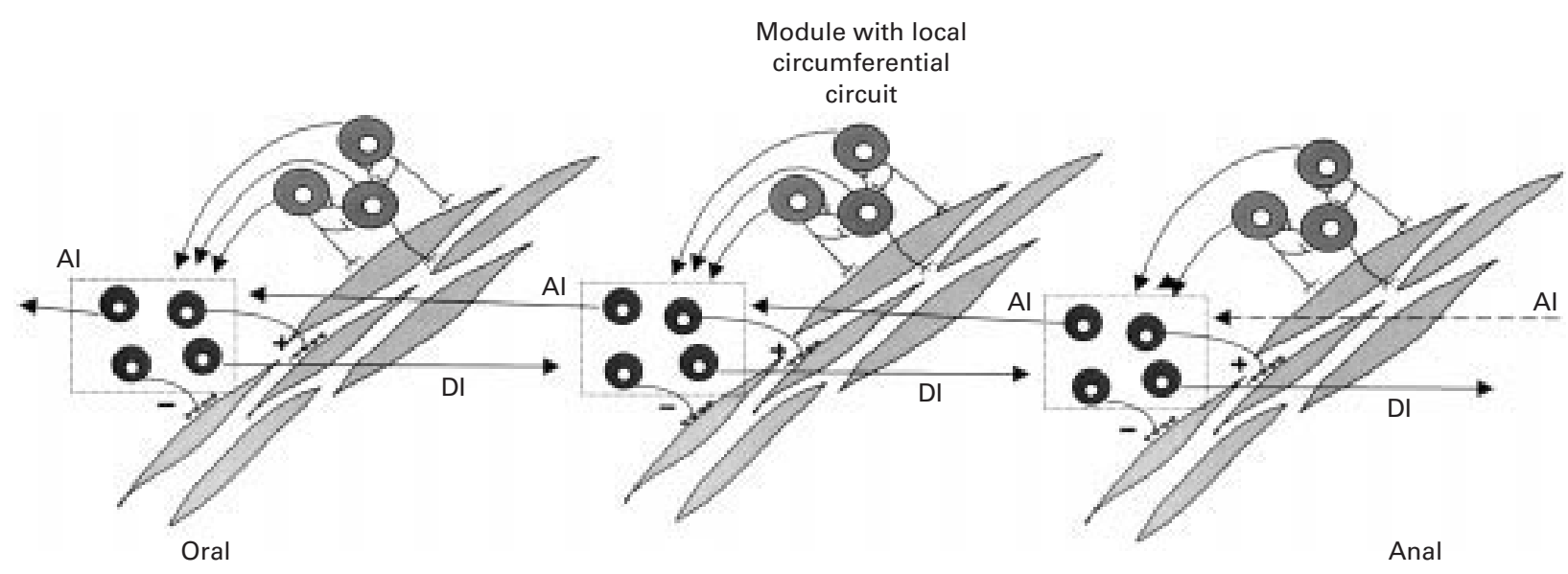

Figure 3 Modular organisation of the enteric nervous system with repeated and overlapping circumferential, ascending, and descending neural pathways. AI, ascending pathways; DI, descending pathways.

cholinergic secretomotor and vasomotor neurones in the submucous ganglia.

MODULAR ORGANISATION OF THE ENS

All classes of enteric neurones are equally distributed along the entire network of ganglia. Thus all enteric pathways described above form continuous overlapping networks. As small rings of circular muscle can contract independently, these rings and the associated enteric neurones can be regarded as functional modules (fig 3). The spatiotemporal coordination of these interconnected modules are the determining factor for the generation of the rich repertoire of motor patterns. ${ }^{24}$

\section{MOTOR PATTERNS}

An important question arises about the role of these reflex pathways in the generation of the different motor patterns. In addition to the myogenic mechanisms that can generate rhythmic contractions and in some cases propulsion, other motor patterns can be distinguished according to their dependence on intestinal contents.

\section{ACCOMMODATION}

Local accommodation is well known to occur in the stomach and large intestine. Also, in the small intestine during filling, the distension activates local pathways, probably a mixture of circumferential and descending inhibitory reflex pathways, that relax the circular muscle. ${ }^{16}$

\section{NEURAL PERISTALSIS}

The propulsion of contents due to the sequential contraction of the circular muscle initiated by the intestinal content has been described as peristalsis. This pattern occurs when the intraluminal volume reaches a threshold or certain chemicals are present in the lumen. This pattern of propulsive behaviour consists of a ring of circular muscle contraction that starts orally and is propagated aborally pushing the contents forward. ${ }^{17}$ The neural circuits involved in this stereotyped motor pattern include activation of the ascending excitatory pathways, descending inhibitory pathways, and possibly activation of descending excitatory pathways. ${ }^{1819}$ It has been suggested, but not confirmed, that in the colon, activation of the collaterals of the extrinsic primary spinal afferent neurones is essential for peristalsis. The possible role of such collaterals of spinal sensory neurones in other motor patterns in normal and physiopathology remains to be elucidated. The exact timing of activation of each of these pathways requires further study. However, it appears that the initiation of peristalsis involves the explosive recruitment of enteric excitatory motorneurones reminiscent of self-reinforcing neural processes. ${ }^{20} 21$ The neurones and mechanisms responsible for this explosive recruitment probably include the primary afferent neurones and the ascending interneurones. Thus peristalsis can be regarded more appropriately as a motor pattern, a type of intestinal locomotion, based on neuromechanical feedback, rather than a simple reflex.

\section{MIGRATING MOTOR COMPLEXES}

One of the most interesting complex neural activities outside of the central nervous system is the neurally mediated migrating motor complex. In addition to the motor patterns which are dependent on the intestinal content activating the enteric reflex pathways (as described above), there are neural circuits within the ENS that spontaneously initiate circular muscle motor activity over extensive portions of the intestine. ${ }^{22}$ The circuits involved in the generation of the migrating motor complex include final excitatory motorneurones, which are activated by other enteric neurones via nicotinic and non-nicotinic mechanisms. As this neural activity slowly migrates aborally, it is probable that some of the descending interneurones are responsible for the aboral migration. Although in the small intestine this cyclic motor activity has been recorded only in conscious animals, evidence suggests that more localised migrating motor activity also occurs in isolated preparations of small and large intestine. It is possible that the segmenting motor pattern described by earlier investigators may be due to more localised, slowly migrating neural activity and its interaction with myogenic rhythmic activity. 


\section{Overview}

The mechanisms of motor activity include myogenic rhythmic activity, with its own spatiotemporal patterns, the content dependent neural mechanisms, namely accommodation and propulsion, and the spontaneous cyclical migrating motor complexes. These mechanisms interact to produce the rich repertoire of intestinal movements. The way in which the relative importance of each of these mechanisms shifts continuously, thus providing an adaptive switching between different motor patterns, is still far from being understood. Yet, studies of this kind will provide the basis for understanding of the physiopathology so often associated with gastrointestinal functions. The muscular and neuronal mechanisms described above are based on rather robust processes. However, there is a wealth of substances contained in the enteric neurones capable of modulating the activities of the enteric neural circuits and of the muscular apparatus. $^{23}$ Every mechanism described is potentially subject to intrinsic physiopathological modulation and is thus a potential target for pharmacological intervention. This opens an enormous new field of research both in gastrointestinal physiology and in the pharmaceutical development of therapies to correct deficiencies and abnormalities.

1 Furness JB, Costa M. The enteric nervous system. Edinburgh: Churchill Livingstone, 1987.

2 Costa M, Brookes SJ. The enteric nervous system. Am $\mathcal{f}$ Gastroenterol 1994;89:129-37.

3 Costa M, Hennig GW, Brookes SJH. Intestinal peristalsis: A mammalian motor pattern controlled by enteric neural circuits. In: Keihn RMH-WO, Jordan LM, Hultborn H, et al, eds. Neuronal mechanisms for generating locomotor activity. New York: New York Academy of Sciences 1998;860:4644 Cos

4 Costa M, Brookes SJH, Song Z-M. From enteric neurons to peristalsis. In: Fuxe K, Hockfelt T, Olson L, et al, eds. Molecular mechanisms of neuronal communication. WennerGren International Series, vol. 68. Amsterdam: Elsevier, 1996: 89-110.
5 Sanders KM. A case for interstitial cells of Cajal as pacemakers and mediators of neurotransmission in the gastrointestinal tract. Gastroenterology 1996;11:492-515.

6 Costa M, Brookes SJH, Steele PA, et al. Neurochemical classification of myenteric neurons in the guinea-pig ileum. Neuroscience 1996;75:949-67.

7 Furness JB, Kunze WAA, Bertrand PP, et al. Intrinsic primary afferent neurons of the intestine. Prog Neurobiol 1998,54:1-18.

8 Brookes SJH, Song Z-M, Ramsay G, et al. Long aboral projections of Dogiel type II, AH neurons within the myenteric plexus of the guinea-pig small intestine. $f$ Neurosci plexus of the guin

9 Brookes SJH, Steele PA, Costa M. Identification and immunohistochemistry of cholinergic and non-cholinergic circular muscle motor neurones in the guinea pig small intestine. Neuroscience 1991;42:863-78.

10 Steele P, Brookes S, Costa M. Immunohistochemical identification of cholinergic neurons in the myenteric plexus of guinea-pig small intestine. Neuroscience 1991;45:227-39.

11 Brookes SJH, Costa M. Enteric motor neurones. In: Tache Y, Wingate DL, Burks TF, eds. Innervation of the gut. Pathophysiological implications. Boca Raton, Florida: CRC Press, 1994:237-48.

12 Brookes SJH, Song Z-M, Steele PA, et al. Identification of motor neurones to the longitudinal muscle of the guinea-pig ileum. Gastroenterology 1992;103:961-73.

13 Brookes SJH, Meedeniya ACB, Jobling P, et al. Orally projecting interneurones in the guinea-pig small intestine. $\mathcal{f}$ Physiol 1997;505.2:473-91.

14 Song ZM, Brookes SJH, Costa M. Projections of specific morphological types of neurons within the myenteric plexus of the small intestine in the guinea-pig. Cell Tissue Res 1996;285:149-56.

15 Bayliss WM, Starling EH. The movements and innervation of the small intestine. F Physiol Lond 1899;24:99-143.

16 Waterman SA, Tonini M, Costa M. Accommodation mediated by enteric inhibitory reflexes in the isolated guinea-pig small intestine. F Physiol 1994;474:539-46.

17 Hennig GW, Costa M, Chen BN, et al. Quantitative analysis of peristalsis in the guinea-pig small intestine using spatiotemporal maps. F Physiol 1999;517:575-90.

18 Waterman SA, Costa $M$. The role of enteric inhibitory motoneurons in peristalsis in the isolated guinea-pig small intestine. F Physiol 1994;477.3:459-68.

19 Waterman SA, Tonini $M$, Costa $M$. The role of ascending excitatory and descending inhibitory pathways in peristalsis in the isolated guinea-pig small intestine. $\mathcal{F}$ Physiol in the isolated

20 Tonini M, Costa M, Brookes SJH, et al. Dissociation of the ascending excitatory reflex from peristalsis in the guineapig small intestine. Neuroscience 1996;73:287-97.

1 Brookes SJH, Chen BN, Costa M, et al. Initiation of peristalsis by circumferential stretch of flat sheets of guinea-pig ileum. F Physiol 1999;516:525-38.

22 Sarna SK. Cyclic motor activity; migrating motor complex. Gastroenterology 1985;89:894-913.

23 Furness JB, Bornstein JC, Murphy R, et al. Roles of peptides in transmission in the enteric nervous system. Trends Neurosci 1992;15:66-71. 